EPiC Series in Engineering
Volume 3, 2018, Pages 2067-2072
HIC 2018. 13th International
Conference on Hydroinformatics

\title{
Estimation of velocity profile in a hyper- concentrated flow: a critical analysis of Bagnold equation
}

\author{
Donatella Termini ${ }^{1 *}$ and Antonio Fichera ${ }^{2 \dagger}$ \\ ${ }^{1}$ Department of Civil, Environmental, Aerospatial and of materials Engineering (DICAM) - \\ University of Palermo, 90128 Palermo, Italy; \\ ${ }^{2}$ Faculty of Engineering, University Enna Kore, Enna, Italy. \\ donatella.termini@unipa.it, antonio.ficheraeunikore.it
}

\begin{abstract}
Debris flow velocity is an important factor which influences the impact forces and runup. Due to the complexity of the phenomenon, it is difficult to define predictive methodologies. The present work reports some results of an experimental run conducted in order to investigate the velocity and sediment concentration distributions. A modified Bagnold's approach to calculate the vertical distribution of flow velocity is presented.
\end{abstract}

\section{Introduction}

Debris flow occurs especially in steep river reaches and are characterized by extreme propagation rapidity and by the ability to move high quantity of fluid mass. For this reason the propagation of debris flow might determine loss of human life and structural damages.

The estimation of the propagation velocity is important to define predictive procedures.

Some numerical codes (see as an example [1], [2]) to simulate the propagation phenomenon have been developed but they need input parameters which are difficult to estimate. Thus, an accurate calibration procedure should be performed by using field and/or laboratory experimental data.

For this reason, experimental research program has been developed at the Hydraulic Laboratory of Department DICAM (University of Palermo - Italy) in a sloping channel.

The present work reports some results of an experimental run conducted in order to investigate the flow velocity and sediment concentration distributions. This information is used to analyze the simplifying hypotheses of the Bagnold's approach. In fact, among the existing theories allowing to

\footnotetext{
* Department of Civil, Environmental, Aerospatial and of materials Engineering (DICAM) - University of Palermo, 90128 Palermo, Italy

${ }^{\dagger}$ Faculty of Engineering, University Enna Kore, Enna, Italy
} 
relate the flow velocity, $u$, to particle concentration, $C$, the Bagnold's (1954) one is the most cited. The Bagnold's [3] equation can be written in the following form:

$$
u=\frac{2}{2 d_{p}}\left[\frac{g \cos \theta}{a_{i} \cos \alpha_{i}} C\left(1-\frac{\rho}{\sigma}\right)\right]^{1 / 2} \frac{1}{\lambda}\left[h^{\frac{3}{2}}-(h-z)^{\frac{3}{2}}\right]
$$

with $\lambda=C^{1 / 3} /\left(c^{* 1 / 3}-C^{1 / 3}\right), u$ is the local mean velocity of flow toward $x$ direction, $d_{p}$ is the particle diameter, $g$ is the acceleration due to gravity, $\theta$ is the channel bed or flow surface slope, $C$ is the particle concentration in volume, $h$ is the local mean depth of flow, $z$ is the height measured perpendicular to the bottom, $\rho$ is the fluid density, $\sigma$ is the particle density, $a_{i}$ end $\alpha_{i}$ are the shape coefficients end $c^{*}$ is the maximum concentration.

Eq.1 is based on the assumption that the concentration particle $C$ is constant along the vertical and the value of $c^{*}$ is uniquely defined. But, literature [4] shows that the concentration varies along the vertical and assumes different values in time.

Thus, in the present work, the data collected during the experimental campaign are used to explore the applicability of the Bagnold's equation [3] by removing the hypothesis of constant sediment concentration.

\section{Material and methods}

The experimental apparatus is constructed at the Hydraulic Laboratory of the Department DICAM - University of Palermo, Italy. The experimental runs were conducted in channel reach $2 \mathrm{~m}$ long and $0.17 \mathrm{~m}$ wide. The bed, with slope is $\mathrm{S}=15^{\circ}$, was of gravel sediments (sediment median diameter $D_{50}=3$ $\mathrm{mm}$ ) of thickness $10 \mathrm{~cm}$ (Figure 1). The side walls of the channel are of Plexiglas. The hyperconcentrated flow was generated by releasing the fixed water discharge $(3.5 \mathrm{l} / \mathrm{s})$ over the mobile gravel bed, preliminary saturated $\left(\mathrm{Q}_{\mathrm{sat}}=0.8 \mathrm{l} / \mathrm{s}\right)$.

The surface concentration value was measured by using a sample of the mixture taken during the hyper-concentrated flow propagation. It was obtained a value of surface concentration equal to $\mathrm{C}=$ 0.305. During the experimental run, the flow velocity and the sediment concentration profiles were obtained by applying the image analysis technique. The images were acquired through a highresolution camera (300 fps was used in the experimental run presented in this work) on the right sidewall of the channel.

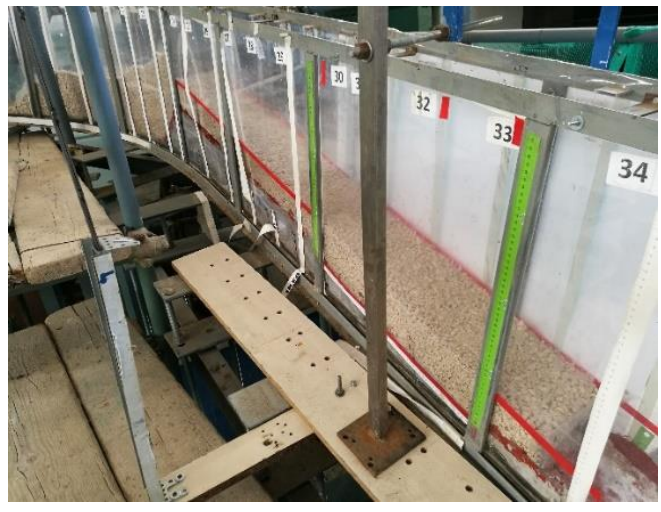

Figure 1: Experimental apparatus. 


\section{Results and discussion}

The acquired images were processed by using a numerical code developed in Matlab environment which allowed us to estimate the vertical profiles both of flow velocity and of sediment concentration at different time instants.

Both from the direct analysis of all the examined frames and as result of the aforementioned procedure, it was verified that the particle concentration varies along the vertical.

In order to estimate the sediment concentrations, the investigated layer was divided into sublayers of thickness $t s$; for each sublayer the average value of sediment concentration was estimated. In particular, the analysis has been conducted for three values of $t s: t s=\mathrm{D}_{50}(3 \mathrm{~mm}), t s=2 \mathrm{D}_{50}(6 \mathrm{~mm})$, $t s=3 \mathrm{D}_{50}(9 \mathrm{~mm})$. Figure 2 shows the experimental concentration distribution estimated for each considered values of thickness $t s$ and Table 1 reports the corresponding value of the root mean square error estimated as follows:

$$
\sigma=\sqrt{\frac{\sum_{i=1, N}\left(C_{i, m}-C_{i, e}\right)^{2}}{N t s}}
$$

$C_{i, m}$ and $C_{i, e}$ are respectively the measured and the estimated values of concentration and Nts is the number of layer of thickness $t s$. From Figure 2 it can be observed that for all the considered values of $t s$ the concentration linearly varies from the value $C^{*}$ (that is the maximum packing concentration) at the bed, to the value of concentration $C_{s}$ at the surface. Furthermore, Table 1 indicates that the values of the root mean square error are always low. This suggests that the linear pattern of concentration does not depend on the layer thickness.

Thus, the analysis of the collected data has allowed us to verify that the experimental vertical distribution of sediment concentration is well approximated by a linear variation between the value of maximum concentration $C^{*}$ at the bed and the measured value at the free surface.

\begin{tabular}{c|c}
\hline Sublayer Thickness $t$ s & \multicolumn{1}{c}{ sqm } \\
\hline 1D $_{50}$ & 0.0274 \\
\hline 2D $_{50}$ & 0.0222 \\
\hline 3D $_{50}$ & 0.0216 \\
\hline
\end{tabular}

Table 1: Values of the mean square error $\sigma$

On the basis of the obtained result, the velocity profile of the hyper-concentrated flow has been estimated by using the Bagnold's equation (1) but assuming a linear variation of the concentration C between the value of $C^{*}$ at the bed and the value of concentration measured at the free surface. It should be noted that the value of $C^{*}$ can be easily determined as soil parameter by geotechnical considerations; the surface concentration should be locally measured.

Armanini et al. [2] presented a relationship which links the value of the surface concentration to the material inclination slope through the internal friction angle. 
Estimation of Velocity Profile in a Hyper-Concentrated Flow: a Critical ... D. Termini and A. Fichera

a)

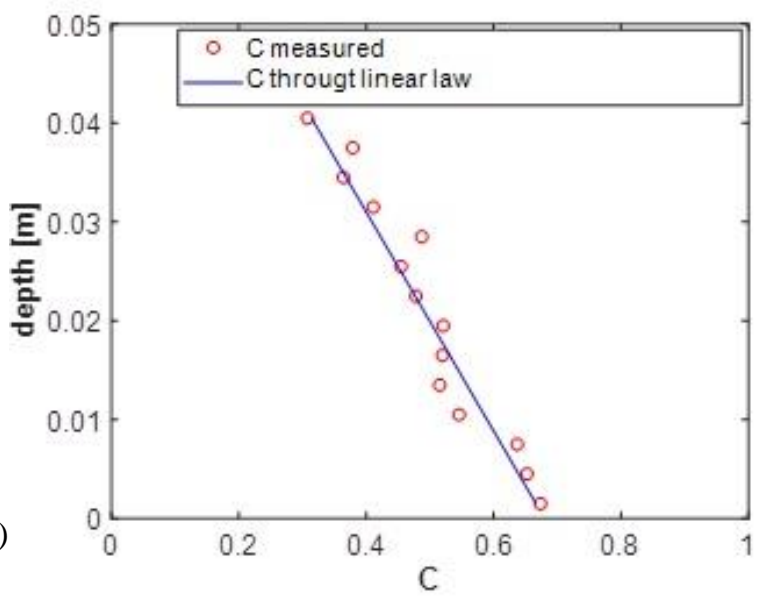

b)
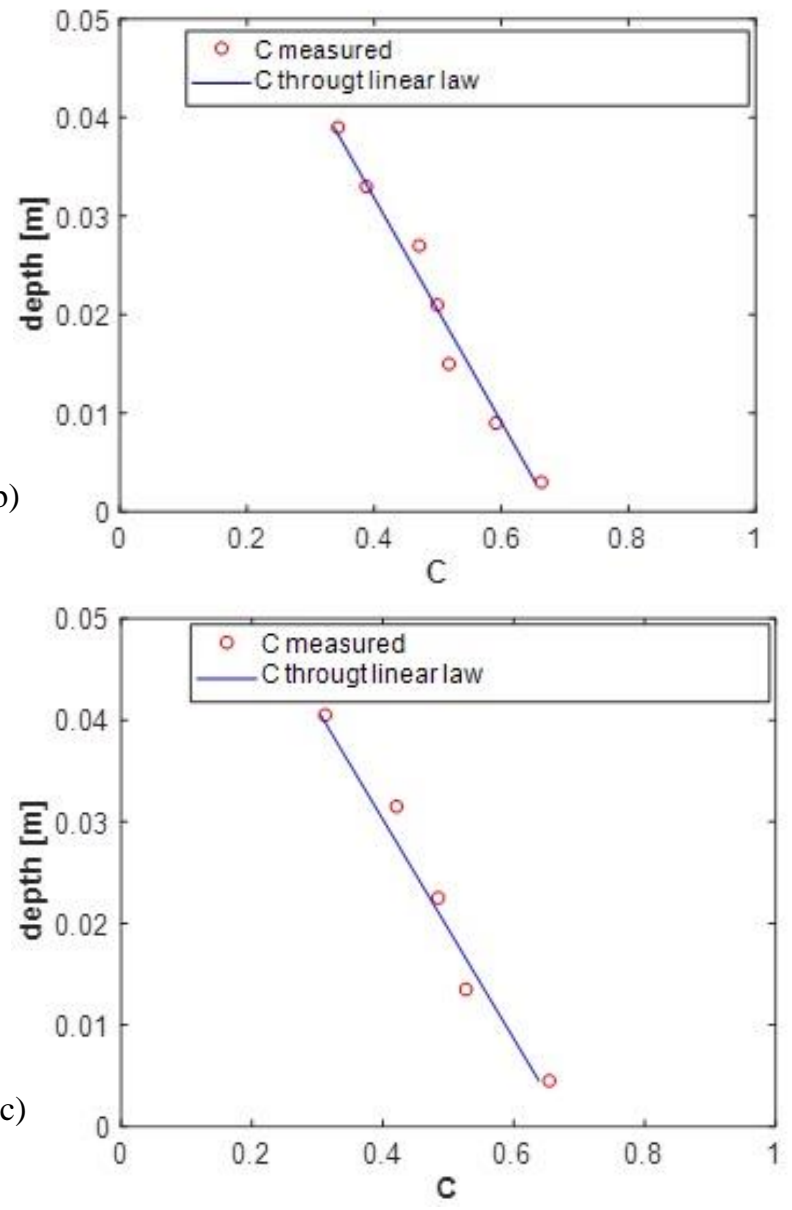

Figure 2: Vertical distribution of sediment concentration: a) $t s=\mathrm{D}_{50}$; b) $t s=2 \mathrm{D}_{50}$; c) $t s=3 \mathrm{D}_{50}$. 
Estimation of Velocity Profile in a Hyper-Concentrated Flow: a Critical ... D. Termini and A. Fichera

Thus, for the presented analysis, the internal friction angle of gravel material used for the experiment has been determined by a direct simple shear apparatus with a large shear box $(30 \mathrm{~cm} \times 30$ $\mathrm{cm} \times 20 \mathrm{~cm}$ ). It has been verified that the value of the internal friction angle obtained as result of the shear tests is almost equal to that obtained by applying the expression presented by Armanini et al. [2] by using the measured value of surface concentration. This procedure allowed us to verify that the expression presented by Armanini et al. [2] could be used to estimate the surface concentration once the internal friction angle of the bed material is known.

In Figure 3 the experimental profile of flow velocity is compared with the $u$-values estimated through equation (1) both by assuming the concentration $C$ constant along the vertical and by considering the linear variation law of the concentration $C$.

From Figure 3 it can be seen the remarkable difference between the $u$-values calculated by assuming $C$ constant and those calculated by assuming $C$ linearly variable. In particular, only the latter well approximates the experimental $u$-values distribution.

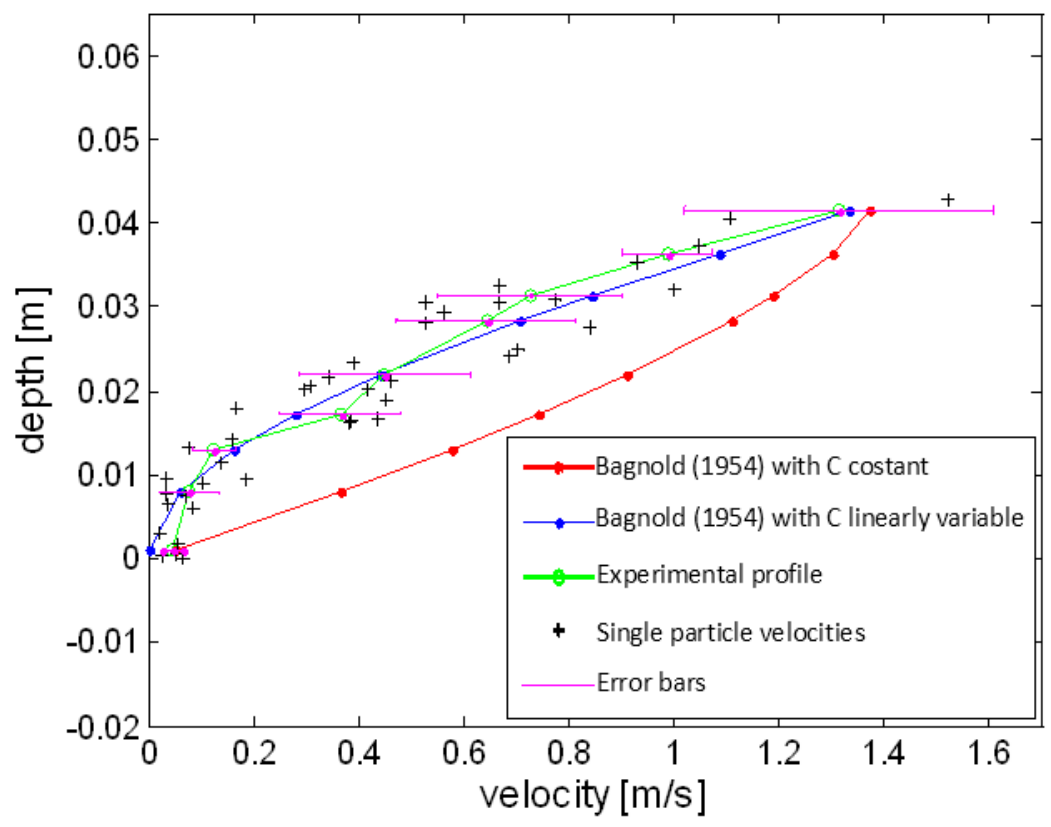

Figure 3: Comparison between the experimental profile and the profiles obtained by Eq. 1 with $C=$ constant and with $C=$ linearly variable. 


\section{Conclusion}

The present work deals with the analysis of the dynamic behavior of a hyper-concentrated flow focusing attention on the velocity distribution.

The data collected during an experimental campaign have been used to investigate the velocity and sediment concentration profiles during the propagation phenomenon. As result, it has been verified that the sediment concentration distribution follows a linear variation law assuming the value of maximum concentration at the bed. This result confirms that the concentration is not constant along the vertical, but it can be considered linearly variable between the two values of sediment concentration: the value of maximum packing concentration (at the bed) and the value of concentration detected on the free surface.

Based on this result, the Bagnold's equation has been used by removing the hypothesis of constant sediment concentration and the velocity profiles have been calculated by assuming the linear variation law of sediment concentration. The comparison between the estimated values with the measured ones has assessed the goodness of the procedure.

\section{References}

[1] O'Brien, J. S., (1986). Physical process, rheology and modeling of mudflows. PhD thesis, Colorado State University, Fort Collins, Colorado, 172 pp., 1986.

[2] Armanini A., Capart H., Fraccarollo, L., Larcher, M., 2005: Rheological stratification in experimental free-surface flows of granular-liquid mixtures. J Fluid Mech 532, 269-319.

[3] Bagnold R. A., 1954: Experiments on a gravity-free dispersion of large solid spheres in a Newtonian fluid under shear. Proceedings of the Royal Society of London A 225: 49-63.

[4] Iverson R. M., 1997: The physics of debris flows, Rev. Geophys. 35 pp. 245-296. 